\title{
Editorial
}

\section{Data Mining and Knowledge Discovery in Industrial Engineering}

\author{
Jun Zhao, ${ }^{1}$ Witold Pedrycz, ${ }^{2,3}$ and Sabrina Senatore ${ }^{4}$ \\ ${ }^{1}$ Dalian University of Technology, Dalian 116023, China \\ ${ }^{2}$ Department of Electrical and Computer Engineering, University of Alberta, Edmonton, AB, Canada T6G $2 G 7$ \\ ${ }^{3}$ Systems Research Institute, Polish Academy of Sciences, 01-447 Warsaw, Poland \\ ${ }^{4}$ University of Salerno, 84081 Baronissi (Salerno), Italy
}

Correspondence should be addressed to Jun Zhao; zhaoj@dlut.edu.cn

Received 12 June 2013; Accepted 12 June 2013

Copyright (C) 2013 Jun Zhao et al. This is an open access article distributed under the Creative Commons Attribution License, which permits unrestricted use, distribution, and reproduction in any medium, provided the original work is properly cited.

Data mining and knowledge discovery play an increasingly important role in the field of industrial engineering, which becomes highly visible considering currently available vast amounts of data being generated every day. This special issue devoted to data mining and knowledge discovery comprises 11 exciting papers. The three main research areas are meticulously discussed, including data-driven based supervisory learning, unsupervised learning, and fault identification. Furthermore, two of the papers concentrate on the data feature extraction and the computational intelligence based optimization.

First, four of the papers report works in the field of datadriven based supervisory learning. Q. Wu and W. Wang propose a piecewise-smooth SVM for an identification problem by designing a twice continuously differentiable function. A fuzzy neural network based regression model is suggested by T. Chen and R. Romanowski for the job cycle time prediction in a wafer fabrication factory. In the perspective of regression prediction, there are two papers elaborating on this issue. A MIMO relationship model reported by W. Mao et al. describes the dynamic similarity between vibration responses of structure, where the multiple-dimensional SVM and ELM based algorithms are employed. In H. Yang and S. Fong paper, an incremental optimization mechanism for a decision tree is proposed to deal with tree size explosion, overfitting, and imbalanced class distribution.
Two of the papers are devoted to unsupervised learning, especially for the data clustering. The paper by J. Tan and R. Wang designs an SNN similarity based clustering algorithm combined with the structure of graph theory. And, by supplying the clustered and ranked knowledge, C. Hu et al. employ the KaM_CLU algorithm and centre_rank strategy for the model improvement to enhance its efficiency and effectiveness.

Three of the papers deal with essential aspects of fault identification and the data processing in industry. An effective approach to identify the faults of variance shifting in a multivariate process is presented by Y. Shao and C. Hou and Y. Liu et al's paper improves the GTD based data imputation by introducing a Gaussian membership function, which finds its application in the energy data center in steel industry. The paper authored by G. Xiong et al. is concerned with a representation of fuzzy knowledge and a realization of reasoning mechanisms for fault diagnosis in power system by using a graphical model.

Y. An et al. extract the geometric features from 3D point cloud data to study the corresponding similarity indicators by using the behaviors of chord lengths, angle variations, and principal curvatures. Y. Li and N. Tan report on a selfadapting Computational Intelligence aimed at developing an optimization task along with its solution to the fleet assignment problem. 
By bringing forward this special issue, we hope the readers will find these studies interesting and highly stimulating.

Jun Zhao

Witold Pedrycz

Sabrina Senatore 


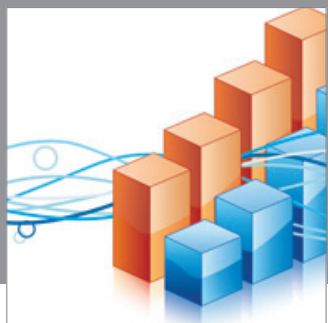

Advances in

Operations Research

mansans

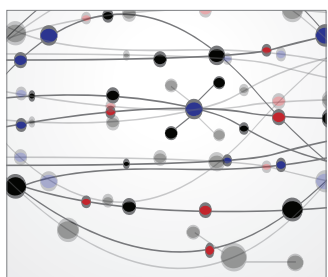

The Scientific World Journal
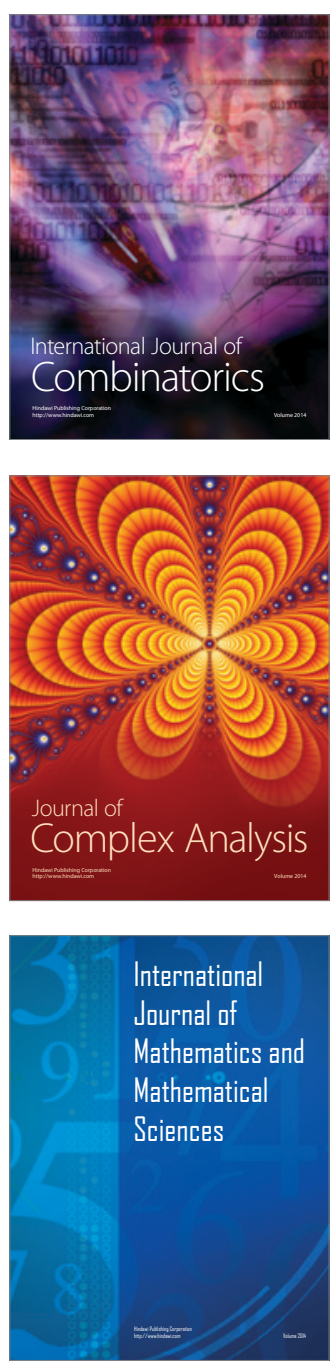
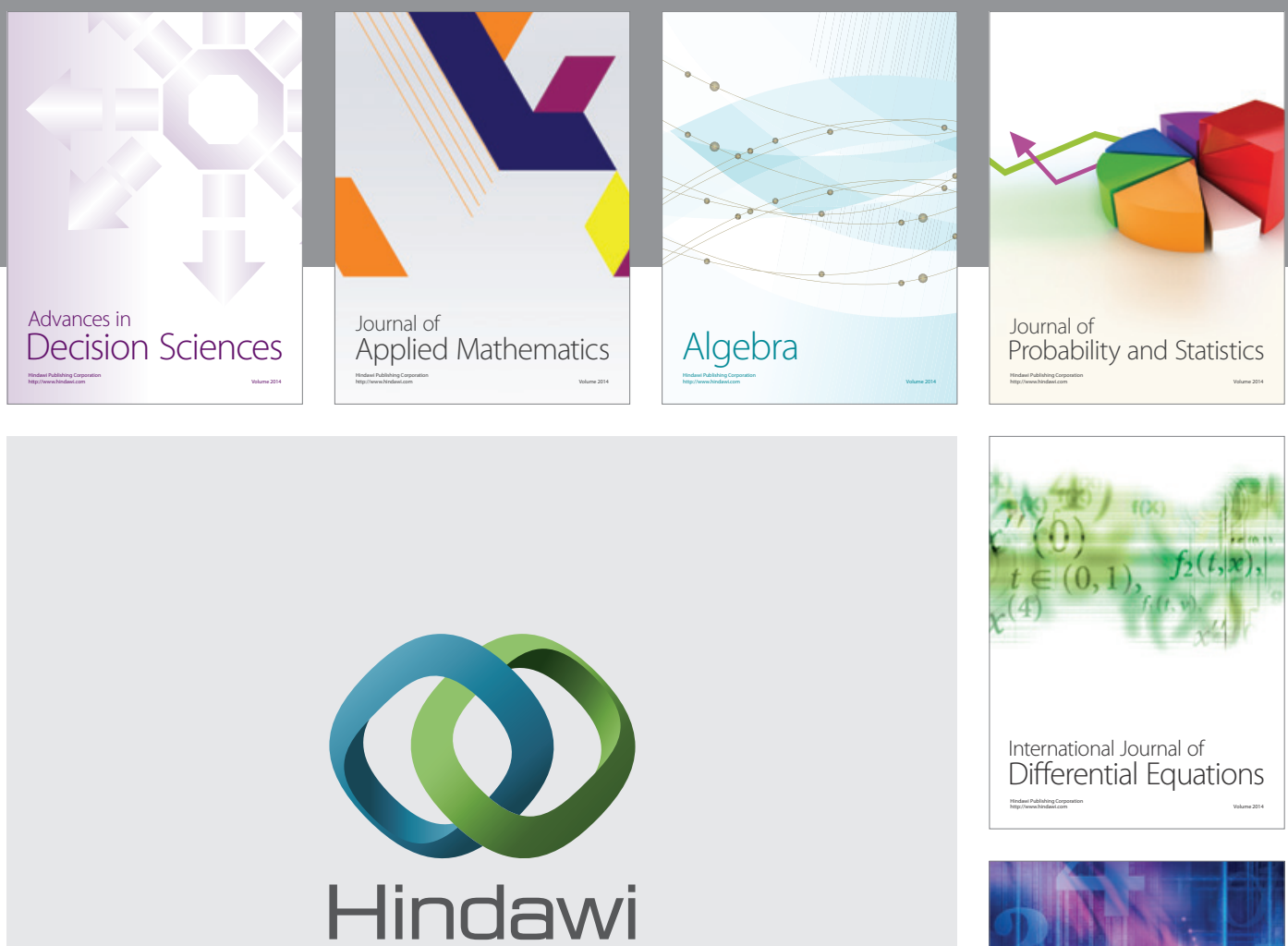

Submit your manuscripts at http://www.hindawi.com
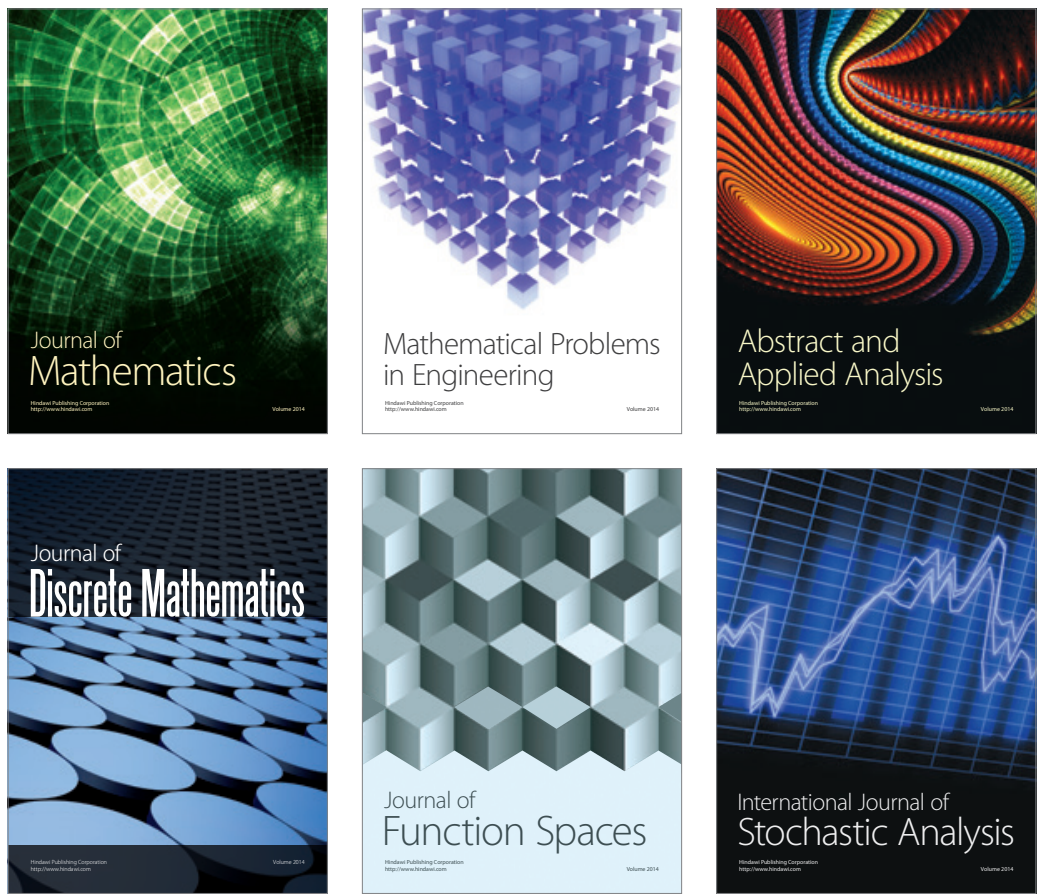

Journal of

Function Spaces

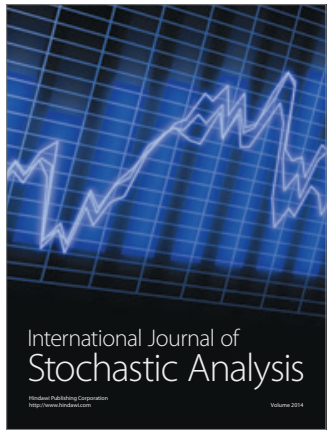

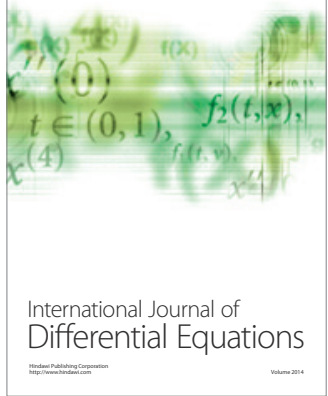
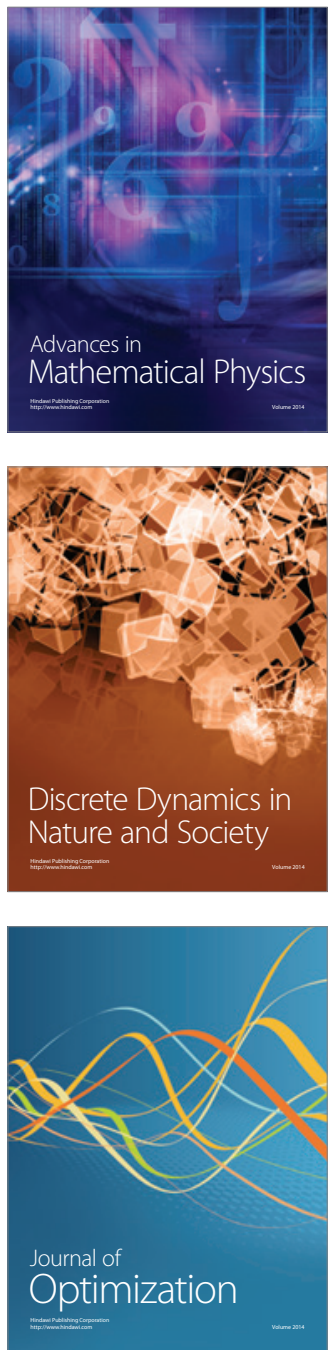